\title{
Retour sur la \\ jurisprudence \\ Perruche
}

Thierry Pech

$>$ La jurisprudence Perruche a fait, plus d'un an durant, l'objet de critiques virulentes. Certaines d'entre elles tiennent à une méconnaissance du droit, de son mode de fonctionnement et de sa nature, d'autres aux effets sociaux de la décision judiciaire. Aux premières, il s'agit ici d'opposer une réponse juridique sur trois points litigieux : le régime de causalité, le droit d'ester de l'enfant et le prétendu «droit de ne pas naître ». Aux secondes, la réponse ne peut être que politique. Celle qui est défendue ici diffère de beaucoup de la position adoptée par le législateur il y a quelques mois : elle propose un dispositif qui consiste à individualiser la responsabilité et à socialiser la réparation, le tout afin de concilier vulnérabilité du praticien et vulnérabilité de la victime. <

Peut être indemnisé du fait de son handicap congénital l'enfant dont la mère a été privée de la possibilité de recourir à une interruption médicale de grossesse en raison d'une faute médicale, alors même que ce handicap était probable et prévisible. Tel était le sens de l'arrêt Perruche, l'une des décissions parmi les plus discutées de la Cour de cassation depuis plusieurs années. Cet arrêt prononcé au mois de novembre 2000 a en effet donné lieu à une longue controverse où ne manquèrent ni les contributions de fond [1], ni les extrapolations et les dérapages inhérents à la polémique. On parla ainsi de « délire d'imputation » ou encore «d'Hiroshima métaphysique ».

Les décisions rendues par la Cour de cassation un an plus

Cet article a fait l'objet d'une précédente publication dans la revue Esprit (janvier 2002). II a été actualisé et modifié pour publication dans médecine/sciences.

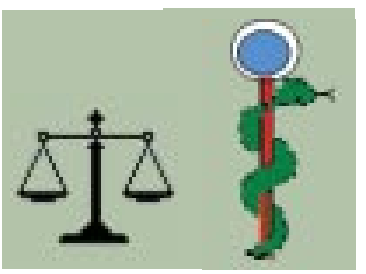

tard dans des affaires comparables n'ont pas manqué de raviver les passions. Si elles ne modifiaient guère le fond du problème, elles s'inscrivaient dans un contexte social et politique beaucoup plus tendu : la virulence d'un certain nombre d'associations, la colère d'une partie du monde médical, les propositions législatives avancées dans la précipitation rendirent plus urgent le réexamen de cette jurisprudence. Les critiques portaient, d'une part, sur le fond de l'arrêt, de l'autre, sur ses effets sociaux à plus ou moins long terme. S'il faut les reprendre ici successivement, ce n'est pas pour refaire le procès hors-les-murs, mais pour tenter de débrouiller les logiques juridique, politique et sociale que le débat a malheureusement trop souvent mélangées. Or, à notre sens, la solution des difficultés soulevées par cette affaire ne relevait pas de la justice - qui a fait ce qu'elle pouvait faire en la matière -, mais plus certainement d'une politique sociale qui fut la grande absente de cette controverse.

D'aucuns pourraient penser que ces questions ne sont plus d'actualité : le législateur les a en effet tranchées cet hiver en adoptant une loi qui annule tous les effets de la jurisprudence Perruche ${ }^{1}$. Mais le débat n'est pas clos pour autant. D'une part, parce qu'en instituant un régime de responsabilité d'exception pour les praticiens concernés et en renvoyant les plaignants à la solidarité nationale et à ses prestations forfaitaires, cette loi frustre un certain nombre de demandes légitimes qui risquent de revenir

1. D'abord, par l'adoption en première lecture à l'Assemblée nationale de la proposition de loi Mattéi amendée par le gouvernement, puis par un nouvel amendement en commission mixte paritaire. 
sous une autre forme sur le devant de la scène. D'autre part, parce que le «problème Perruche » interroge certaines de nos pratiques institutionnelles très au-delà du seul cas des enfants nés handicapés à la suite d'une erreur de diagnostic : outre qu'il souligne à nouveau les difficultés du débat public à appréhender les décisions judiciaires dans leur registre de pertinence, il met au défi d'inventer de nouveaux dispositifs de socialisation des risques liés au développement des disciplines prédictives.

\section{Les critiques juridiques de l'arrêt Perruche}

Le cas qui est à l'origine de l'arrêt Perruche mérite d'être brièvement rappelé. Mme Perruche, enceinte d'environ un mois, craint d'être atteinte d'une rubéole. Consciente des risques de malformations encourus de ce fait par l'enfant qu'elle porte (syndrome de Gregg), elle est engagée par son médecin à procéder à des examens sérologiques et annonce, avant d'en connaître les résultats, que s'ils s'avéraient inquiétants, elle aurait recours à une interruption volontaire de grossesse. Les trois examens successifs qui sont réalisés conduisent à des conclusions rassurantes.

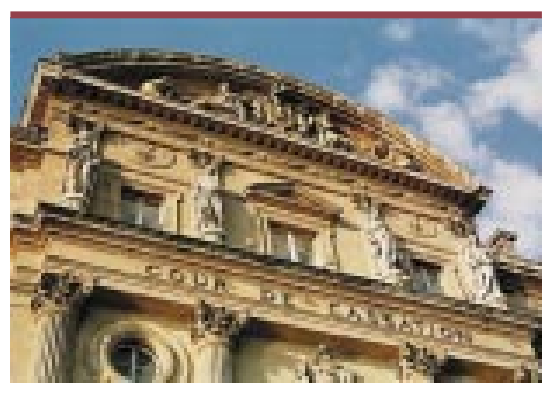

Cour de Cassation.
Or, l'enfant qui vient au monde huit mois plus tard présente presque toutes les manifestations du syndrome de Gregg : troubles neurologiques graves, surdité bilatérale, cécité de l'œil droit, glaucome, cardiopathie impliquant en permanence l'assistance d'une tierce personne. S'engage alors une procédure judiciaire qui, des premières expertises démontrant la faute médicale jusqu'à la décision finale de la cour de cassation, durera une quinzaine d'années. Un long parcours au terme duquel la justice estimera : (1) que la responsabilité du médecin et du laboratoire est engagée ; (2) que la mère doit être indemnisée au titre de la « perte d'une possibilité d'avorter» (aspect de l'affaire qui ne fut guère contesté et que nous laisserons de côté); (3) que l'enfant lui-même doit être indemnisé du fait de son handicap.

C'est sur cette dernière disposition qu'a porté l'essentiel du débat juridique ${ }^{2}$. Au terme d'une campagne de presse d'une intensité inhabituelle compte tenu du caractère très technique de son objet, il est apparu que la tendance majoritaire dans l'opinion était plutôt au désaveu de la décision rendue par la Cour de cassation. Un désaveu soutenu par de nombreux juristes qui concentrèrent leurs attaques sur trois points dont cer- tains flirtent avec des considérations d'ordre métaphysique : (1) le lien de causalité entre l'erreur et le préjudice ; (2) le droit d'agir en justice de l'enfant ; (3) la dénonciation du prétendu « droit de ne pas naître ».

\section{Le lien de causalité entre la faute médicale et le préjudice}

L'affaire Perruche ne doit pas être située dans le cadre de la responsabilité pénale (il n'est pas question de rechercher une infraction et un coupable à punir), mais dans celui de la responsabilité civile: il s'agit de rechercher la réparation d'un préjudice. Ou plus exactement dans le cadre de la responsabilité contractuelle du dommage causé à un tiers ${ }^{3}$. La nature de ce contrat consiste dans le lien qui unissait la mère enceinte atteinte de la rubéole au médecin et au laboratoire d'analyses médicales chargés d'établir le diagnostic et de satisfaire ainsi leur obligation d'information et de conseil à l'égard de la patiente. Le tiers à cette relation contractuelle, c'est le futur enfant, à l'époque le fœtus. Mais, pour établir la responsabilité du laboratoire d'analyses médicales et du médecin dans cette affaire, il faut d'abord pouvoir démontrer qu'il existe un lien de causalité entre l'inexécution de l'obligation d'information et le dommage causé. Ce lien de causalité existe-til ? Non, répondent les détracteurs de l'arrêt qui situent la causalité dans le registre des causes naturelles et prépondérantes. La cause objective du dommage, disent-ils, c'est la rubéole de la mère. Le médecin ne peut être considéré comme la cause de cette pathologie qui préexiste à son intervention. De fait, la cause directe du préjudice n'est pas l'erreur de diagnostic. Comment s'opposer à une telle évidence? D'ailleurs l'arrêt de la Cour de cassation ne s'y oppose pas : il ne dit pas que la rubéole de la mère n'est pas une des causes du préjudice. II ne dit même pas que ce n'est pas la cause prépondérante. II dit seulement que ce n'est pas la seule et qu'il existe des causes secondaires qui doivent être prises en compte. Car le droit conçoit plusieurs théories de la causalité : la théorie dite de la causalité adéquate (on ne retient que la cause prépondérante, ici : la rubéole de la mère) ; la théorie dite de la proximité des causes (seule la dernière cause est retenue); la théorie dite de l'équivalence des conditions (tout fait, fût-il éloigné, sans lequel

\footnotetext{
2. En effet, la question de la nature des fautes commises par les praticiens, et notamment de leur niveau de gravité, n'a guère été débattue devant la Cour de cassation. Elle est intervenue plus tard, dans le débat public, à l'initiative des gynécologues et des échographistes en particulier. Nous y reviendrons.

3. C'est l'arrêt Mercier (1936) qui a, pour la première fois, défini ce cadre : « [...] il se forme entre le médecin et son client un véritable contrat comportant pour le praticien l'engagement sinon, bien évidemment, de guérir le malade, [...] du moins de lui donner des soins [...] consciencieux, attentifs et, réserve faite de circonstances exceptionnelles, conformes aux données acquises de la science ».
} 
le dommage ne se serait pas produit, est réputé causal). En vertu de cette dernière théorie, on peut donc dire : rien de tout cela n'aurait eu lieu si le médecin et le laboratoire d'analyses n'avaient commis aucune erreur, car la mère aurait avorté (c'est ce qu'elle avait affirmé avant l'examen) si elle avait été correctement informée. La mère a donc été, non seulement privée d'une « chance d'avorter », mais du même coup d'un choix qui aurait peut-être permis d'éviter le dommage. II existe donc, pour la cour, un lien de causalité entre l'erreur de diagnostic et le handicap de l'enfant.

Cette position appelle quelques commentaires tant elle a suscité l'indignation, notamment de la part des médecins. La causalité telle que déduite de la théorie de l'équivalence des conditions est certes en complet décalage par rapport à la causalité biologique considérée par ceux-ci comme suffisante, mais elle reste très utile quand on cherche, non à déceler la cause directe et naturelle d'un phénomène, mais aussi à solidariser des chaînes de décisions complexes. Elle se déploie dans l'ordre d'une finalité poursuivie collectivement autant que dans l'ordre des faits observables : c'est un «pour» autant qu'un «parce que ». Or, plus la technologie dilue les responsabilités de chacun dans une longue série de gestes et de choix qui peuvent aboutir à des conséquences catastrophiques, plus on sera tenté d'étendre le champ d'application de la responsabilité individuelle des acteurs pour que chacun d'eux ait conscience qu'il y a des vies humaines en bout de chaîne, des hommes et des femmes au-delà de l'écran, de l'éprouvette ou de la circulaire. L'équivalence des conditions permet ainsi d'appréhender les conditions sociales qui, sans être des causes prépondérantes, ont contribué à la survenue du dommage, et de créer des chaînes de responsabilités où l'on pourrait ne voir qu'une série de choix techniques sans gravité. Sans cette théorie de l'équivalence des conditions, il n'y aurait pas eu d'affaire du sang contaminé, par exemple. Certains s'en féliciteront peutêtre. Mais, sans cette théorie, il pourrait aussi être très difficile d'établir la responsabilité des employeurs dans certains cas d'accidents du travail pour manquement à une obligation de prudence ou de sécurité.

Reste que ce chaînage causal entre l'inexécution de l'obligation d'information et la survenue du dommage via la perte d'une possibilité d'avorter, crée un dispositif particulièrement complexe et délicat. Le point central en est toujours la possibilité donnée à la mère d'exercer un choix éclairé. De fait, dans ce système, si la mère est correctement informée et qu'elle choisit d'avorter, le juge ne voit rien à redire. Si la mère est mal informée et qu'elle donne naissance à un enfant handicapé, le juge peut accueillir favorablement la demande d'indemnisation de la mère et également celle de l'enfant (c'est le cas de l'arrêt Perruche). Mais envisageons le cas d'une mère correctement informée qui choisit en son âme et conscience de donner naissance à un enfant handicapé. Dans ce dernier cas, le juge ne peut absolument pas recevoir l'éventuelle demande d'indemnisation de l'enfant. Autrement dit, l'enfant porte alors pleinement le choix de sa mère, celui-ci étant souverain et correctement éclairé. Cette dissymétrie interdit d'imaginer comme certains l'ont fait que bientôt des enfants pourraient se retourner contre leurs parents du seul fait d'avoir été mis au monde. Mais elle signifie également que le handicap de l'enfant ne peut être évalué de la même manière selon qu'il survient à la suite de l'inexécution de l'obligation d'information ou qu'il a été délibérément accepté par la mère. En ce sens, affirmer que «la force du projet parental de la mère permet à l'enfant de s'en prévaloir comme d'un droit propre à l'encontre du médecin qui l'a empêché de se réaliser $\gg([2]$, p. 194) est insuffisant. II faut compléter cette proposition par une autre, presque inverse : la force du projet parental de la mère empêche l'enfant de se prévaloir d'un droit propre pour obtenir la réparation d'un handicap qu'elle aurait accepté pour lui. Dans un cas, c'est bien « la volonté maternelle qui permet à l'enfant handicapé de se plaindre du préjudice subi » ([2], p. 200), mais, dans l'autre, c'est cette même volonté qui l'en empêche.

\section{La légitimité de l'enfant à agir en justice}

Une autre question est de savoir si l'enfant est fondé à porter plainte en son nom propre en raison du préjudice qu'il a subi alors qu'il n'avait aucune autonomie de choix et qu'il n'était pas encore constitué comme sujet de droit (titulaire de droits et capable d'agir en justice). En termes plus juridiques: «l'enfant handicapé peut-il user de sa qualité de sujet de droit pour demander la réparation d'un préjudice qui résulte du fait de n'avoir pas été avorté par ses parents, ce qui l'aurait empêché de devenir sujet de droit?» [3].

Risquent de s'enchevêtrer ici deux questions qu'il convient de distinguer : le droit d'agir en justice, d'une part, et la question de l'éventuel «droit de ne pas naître », de l'autre. La première question est de savoir si la qualité de sujet de droit peut avoir des effets juridiques rétroactifs portant sur une période où le sujet de droit n'était pas

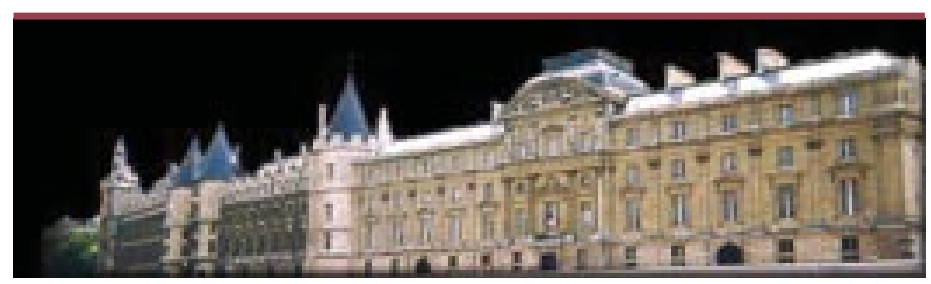

Conciergerie Palais de Justice (Paris) 
constitué. C'est ce qu'affirme la Cour de cassation en l'espèce en accueillant la demande de l'enfant. Est-ce une révolution, comme certains l'ont prétendu?

Une question analogue se pose, par exemple, quand le père d'un enfant conçu décède avant la naissance de celui-ci, c'est-à-dire avant que l'enfant n'accède au statut de sujet de droit. Dans ces conditions, l'enfant peut-il réclamer la succession de son père alors qu'il n'en a pas été le contemporain comme sujet de droit ? Au moment où le père décède, l'enfant n'est pas son héritier puisqu'il n'existe pas encore juridiquement comme sujet de droit. Mais peut-il devenir rétroactivement son héritier ou bien faut-il admettre que l'enfant ne pourra pas hériter de son père ? Dans l'intérêt de l'enfant, le droit crée une fiction : la fiction de l'infans conceptus. Dès lors qu'il est établi que l'enfant est né viable, on fait remonter au moment de sa conception les effets juridiques de sa qualité de sujet de droit en matière patrimoniale de sorte qu'il peut entrer dans la succession d'un père décédé qu'il n'aura jamais connu. Autrement dit, le droit restaure artificiellement, dans l'intérêt de l'enfant, la continuité rompue de la filiation. C'est un processus d'attribution rétroactive et artificielle de la qualité de sujet de droit.

Le cas qui nous occupe est un peu différent, mais il repose au fond sur le même mécanisme. II ne pose pas une question de nature patrimoniale liée à la succession, mais l'intérêt de l'enfant peut jouer en faveur d'une telle fiction. Car il s'agit bien de défendre effectivement l'enfant, comme l'a souligné la cour dans son rapport annuel. Et pour cela, la seule indemnisation des parents était une bien faible garantie : qui peut dire ce qu'il adviendrait des sommes allouées aux parents dans le cas où ceux-ci viendraient à mourir, à se séparer ou à se brouiller avec l'enfant? Pour protéger effectivement celui-ci, il fallait donc pouvoir lui allouer personnellement une indemnisation du préjudice. C'était la seule possibilité pour mettre l'enfant à l'abri des « aléas d'aides familiales privées ou publiques ».

Un second artifice est utilisée dans cette affaire : celui de la représentation.

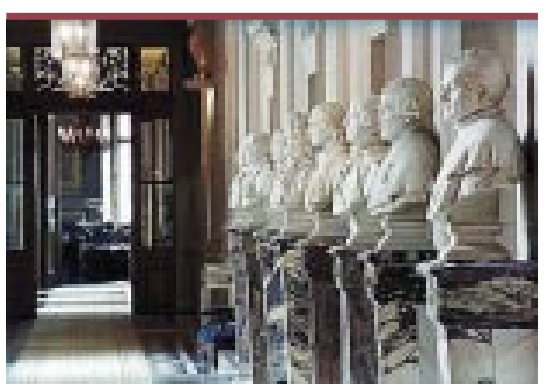

Galerie des bustes de la Cour de Cassation
C'est l'enfant qui porte plainte, mais, compte tenu de son handicap, il ne peut parler que par la voix de ses représentants légaux, en l'occurrence de ses parents. Certains se sont émus de ce mécanisme: pour eux, la plainte de l'enfant n'était en réalité que la seconde plainte de ses parents déjà indemnisés en leur nom propre pour la perte d'une chance d'avorter. De même, le fait de présumer que l'enfant lui-même regarde son handicap comme un préjudice et de le dire en son nom ne va pas sans poser problème. Cependant, présumer le contraire pourrait tomber sous le coup de la même critique. Faut-il pour autant se résoudre à taire une souffrance parce qu'on ne l'endure pas soi-même? Le risque serait grand de condamner au silence un grand nombre de personnes. Renoncer à cette fiction de la représentation, c'est priver les « incapables de droit » de toute capacité à agir en justice et à faire valoir leurs droits personnels. La fiction de la représentation est, à ce jour, le seul moyen connu d'assurer les droits de la défense et de l'action judiciaire à tous les sujets réputés incapables. Incontestablement, il s'agit d'un artifice, mais d'un artifice utile et même essentiel au respect de la dignité humaine, un artifice sans lequel les handicapés mentaux, par exemple, n'auraient aucun moyen d'être juridiquement protégés en tant que personnes. Du reste, les enfants trisomiques défendus récemment par certaines associations comme le «Collectif contre l'handiphobie » ne s'expriment pas moins par représentants interposés. Autrement dit, ce qui choque dans le prétoire deviendrait acceptable dans le débat public... II y a là une contradiction manifeste.

\section{Au sujet d'un prétendu droit de ne pas naître}

Reste la difficile question d'un éventuel «droit de ne pas naître ». Un tel droit serait effectivement absurde car ce serait un droit sans sujet, donc sans exercice, les fœtus n'ayant, jusqu'à preuve du contraire, aucune autonomie de choix. À la limite, il pourrait y avoir un «droit de n'être pas né » puisque le sujet de droit succède nécessairement à sa naissance. Mais un tel droit serait également absurde puisqu'il ne pourrait s'exercer qu'une fois la naissance accomplie. Sauf à imaginer que ce droit vienne valider une demande de mort conçue comme la négation-réparation d'une naissance préjudiciable, hypothèse parfaitement contraire au principe fondamental du respect de la vie humaine. Bref, l'éventuel «droit de ne pas naître » ou « de n'être pas né » relève d'une hypothèse ou insensée ou monstrueuse.

Mais toutes ces spéculations reposent sur l'idée que le préjudice consiste dans la vie même de l'enfant, ce que le raisonnement de la cour récuse : les juges indemnisent le handicap et non la vie. La naissance est en effet le moyen par lequel le dommage est survenu, mais elle ne constitue pas le préjudice en lui-même. C'est parce que l'enfant vit qu'il souffre, mais il ne souffre pas de vivre : il souffre d'un handicap congénital et il y a néanmoins autre chose que ce handicap dans sa vie. L'être qu'on a laissé venir au 
monde handicapé a gagné avec la vie quelque chose qui excède son handicap : la dignité humaine. Ces nuances sont d'importance car il serait contraire aux droits les plus fondamentaux de considérer que la vie puisse en elle-même constituer un préjudice. Cela signifierait clairement que certaines vies ne valent pas d'être vécues, ce qui n'est pas le propos du présent arrêt.

Le préjudice n'est donc pas la vie elle-même, mais le handicap qui lui est attaché et ses conséquences humaines, sociales et économiques. II s'agit de réparer un préjudice qui est là, non de spéculer sur ce qui aurait pu être. La cour ne dit pas qu'il eût été préférable que l'enfant ne vînt pas au monde - même si ç'eût été pratiquement le seul moyen d'éviter la survenue du dommage. Elle dit : il y a un dommage qui est lié de manière causale à l'erreur de diagnostic. Encore une fois, le droit ne se situe pas seulement dans une logique du constat, mais aussi dans une logique d'action qui cherche à régler des relations, à équilibrer des intérêts, à rendre le monde plus vivable. La justice n'est pas réductible à une vérité, fût-elle scientifique : elle consiste aussi dans une action et dans une volonté.

Cette logique conduit le juge à prendre acte du fait que l'enfant vit, qu'il est désormais un être social et qu'il faut en assumer toutes les conséquences. Du reste, la demande de Nicolas Perruche n'est pas une demande de mort (comme dans le cas de ce couple anglais dont le mari a demandé à la justice de l'autoriser à tuer sa femme qui, atteinte d'une grave maladie neuro-dégénérative, lui réclame la mort), mais une demande de réparation, d'amélioration d'un sort peu enviable.

On peut considérer que cette dissociation de la vie et du handicap est un artifice juridique supplémentaire puisque dans la réalité du processus qui conduit de l'erreur de diagnostic à la naissance, les deux sont inextricables : on ne peut ni avoir l'un sans avoir l'autre, ni éviter l'un sans nier l'autre. Certes, il s'agit encore d'une fiction, mais - il faut le souligner - d'une fiction qui satisfait une exigence morale fondamentale : cette vie qu'on a laissé venir porte plus que son handicap, il y a en elle quelque chose qui est en excès sur le handicap, quelque chose qui justement vaut d'être vécu et veut être reconnu. Au fond, non seulement l'arrêt ne dit pas que certaines vies ne valent pas d'être vécues, mais il conduit au contraire à considérer que c'est parce que toute vie vaut d'être vécue qu'il faut indemniser Nicolas Perruche. La reconnaissance et la dignité ne sont pas toujours du côté où l'on croit.

\section{La critique des effets sociaux de l'arrêt}

Ce rapide examen des critiques juridiques qui ont été opposées à la jurisprudence Perruche, souligne la cohérence du raisonnement des juges et dissipe un certain nombre d'inquiétudes éthiques. Les juristes qui ont pris la défense de l'arrêt incriminé ont en général insisté sur ce point. Malheureusement, ils se sont le plus souvent arrêtés là, s'enfermant dans la rationalité du droit et négligeant de répondre

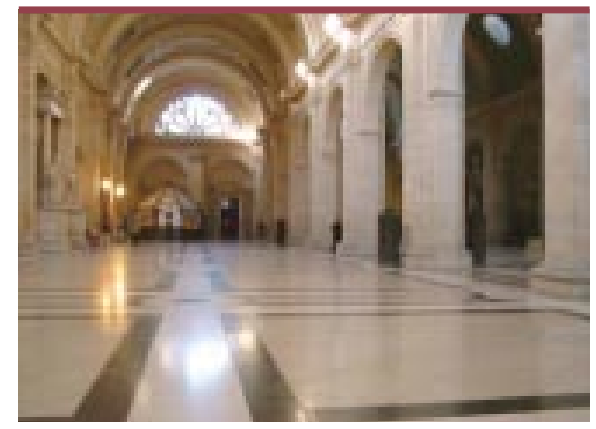

Salle des Pas Perdus du Palais de Justice aux autres questions soulevées par «l'affaire Perruche $»^{4}$. Or, celle-ci a également fait l'objet de critiques qui mettaient en exergue les effets désastreux d'une telle décision, d'une part, sur la médecine prénatale, de l'autre, sur l'image sociale des handicapés. Nombreux sont ceux qui, parmi ces derniers, ont vu dans les arrêts successifs de la Cour de cassation un témoignage de mépris propre à dévaloriser des personnes dont la solidarité nationale exige au contraire la reconnaissance.

Du côté des praticiens de la médecine prénatale, on redoutait surtout que l'inflation du « risque judiciaire » ne conduise certains cabinets à mettre la clé sous la porte et ne dissuadent les plus jeunes de s'engager dans ces spécialités ${ }^{5}$ alors même que la demande de diagnostic ne cesse de croître. Le débat a d'abord tourné autour de la nature des fautes susceptibles d'être raisonnablement reprochées aux médecins : beaucoup de médecins considéraient que le fait de reconnaître comme une faute médicale tout diagnostic erroné consistait à les placer devant une obligation de résultat intenable dans l'état actuel des techniques, voire à les inciter quasi systématiquement à conseiller l'interruption médicale de grossesse, transformant leurs cabinets en antichambres de l'avortement préventif. II faut rappeler à cet égard que la très grande majorité des fautes visées par les différents arrêts incriminés ne résultaient pas d'un manque de fiabilité des techniques de dépistage, mais de comportements professionnels objectivement fautifs, lesquels procédaient d'un manquement caractérisé à leur obligation de moyens ou, plus précisément encore, à leur obligation contractuelle d'information des patients : omission ou refus de prescrire des examens de dépistage qui auraient permis de mesurer un risque d'anomalie, non-respect d'un protocole

\footnotetext{
4. C'est le cas notamment de M. Lacub [2].

5. Il faut rappeler qu'aucun cabinet d'échographie n'a jamais été condamné dans les affaires traitées par la Cour de cassation dans le cadre de la jurisprudence Perruche. La mobilisation de ces praticiens n'avait d'autre raison que la crainte d'être bientôt touchés et l'affolement des assureurs qui auraient été, dans cette hypothèse, les premiers payeurs.
} 
d'examen biologique, absence de réaction du praticien auquel ont été communiqués les résultats alarmants d'un dépistage de la trisomie qu'il avait pourtant luimême recommandé...

Mais le débat a également tourné autour du poids des indemnisations et de l'augmentation insupportable des primes d'assurance ${ }^{6}$. L'enjeu financier de telles affaires n'est pas négligeable. II faut rappeler à cet égard, pour en donner une idée, que, dans la seule affaire Perruche, les sommes réclamées par la famille et la Caisse primaire d'assurance maladie de l'Yonne (qui était partie civile) s'élevaient à plus de $305000 €$. En outre, au regard des assureurs et du calcul actuariel, la décision judiciaire et le niveau d'indemnisation qu'elle fixe restent très aléatoires. Ajoutons enfin que le « risque judiciaire » est très inégal selon que l'on exerce dans le secteur public où l'État assume en dernière instance la charge des réparations, ou dans le secteur privé où c'est aux assurances privées des cabinets d'en couvrir les frais.

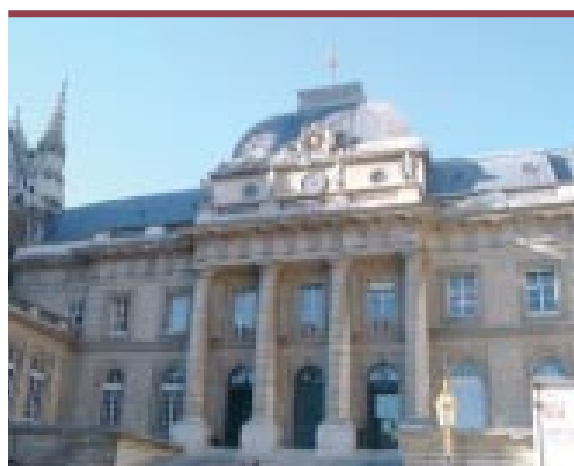

Entrée du Palais de Justice
Les praticiens de la médecine prénatale et les associations d'handicapés opposés à la jurisprudence Perruche se retrouvaient donc unis dans une même lutte. Mais, tandis que celles-ci faisaient valoir l'exigence de reconnaissance et d'acceptation sociale du handicap, le monde médical portait majoritairement sa critique au nom de la défense de disciplines dont la finalité revendiquée consiste précisément dans le dépistage du handicap et l'offre d'une possibilité de le rejeter par l'interruption médicale de grossesse. Le camp des détracteurs de la jurisprudence Perruche n'était donc pas homogène : au contraire, il était traversé de conflits potentiels qui n'ont pas trouvé d'expression publique, mais qui restent latents.

Quoi qu'il en soit, peut-on contester juridiquement le bien-fondé d'une décision de justice au motif qu'elle a des conséquences collectives discutables? Cette interrogation nous porte aux confins de deux logiques distinctes : celle du jugement judiciaire, d'une part, et celle du jugement politique, de l'autre. La première se préoccupe du respect de la loi et des libertés individuelles. La seconde se soucie plus immédiatement de

6. Le montant des primes d'assurance des cabinets privés d'échographie a été multiplié par dix entre novembre 2000 et décembre 2001. l'intérêt général et du plus grand nombre. Or, ces deux logiques ne sont pas nécessairement convergentes. L'affaire qui nous occupe montre à cet égard que le jugement judiciaire peut entrer en contradiction avec le jugement politique. Bref, les décisions de justice ne sont pas nécessairement des décisions de paix sociale, elles peuvent même y être opposées. La condition et la preuve d'une justice indépendante et impartiale consistent notamment dans sa capacité à prendre des décisions impopulaires quand la loi l'exige ou si elle le permet. Ce qui ne signifie pas que les considérations liées à l'effet social des décisions doivent être écartées des motifs du jugement, mais qu'elles n'y sont pas toujours prioritaires, qu'elles ne priment pas le respect de la loi et la protection des droits. Si les effets sociaux de ces décisions sont contestables, alors ce n'est pas au juge, mais au législateur de modifier les lois qui ont autorisé de telles décisions.

Nous sommes ici au cœur d'une tension vive de la vie démocratique, entre, d'une part, la légitimité d'un individu à faire valoir ses droits personnels devant le juge et, de l'autre, la poursuite d'intérêts plus généraux. C'est cette tension qui agite aujourd'hui le monde des associations d'handicapés, partagées qu'elles sont entre la défense de l'acceptabilité sociale du handicap en général et celle des handicapés et de leurs familles qui ont lancé ces actions en responsabilité contre le monde médical. On a ainsi entendu des membres du Collectif contre l'handiphobie $(\mathrm{CCH})$ condamner « ces démarches individuelles contraires à la notion même de solidarité nationale vis-à-vis des handicapés ». Si respectable soit-il, le principe de solidarité peut-il conduire à suspendre des droits aussi fondamentaux que celui d'agir en justice et de réclamer l'arbitrage d'un tiers indépendant et impartial ? Certainement pas : là passe précisément la frontière entre une démocratie respectueuse des droits de l'homme et des régimes de plus sombre mémoire.

Par ailleurs, on peut considérer que la responsabilisation de la médecine prédictive - et en particulier de la médecine prénatale - est en partie le salaire de son succès. Car, grâce ou à cause d'elle, des événements considérés naguère comme des accidents sortent peu à peu du registre de la fatalité et glissent progressivement vers celui de la maîtrise technique. Autrement dit, le progrès scientifique engendre peu à peu de la responsabilité où il n'y avait que du hasard. Les patients en paieront peut-être un jour le prix, mais les praticiens aussi. La fatalité avait les yeux bandés; en faisant savoir, les biotechnologies font assumer : elles lèvent le voile et exigent des acteurs une plus grande responsabilité. La question est de savoir si cette responsabilité doit être individuelle (civile ou pénale) ou bien collec- 
tive, si elle doit conduire à des imputations personnelles ou à de nouveaux mécanismes de socialisation des risques. Cette question, hélas, n'a jamais été posée. D'une part, parce que le politique n'a jamais cherché à la formuler publiquement, préférant suivre l'opinion publique plutôt que de la construire et de la documenter sérieusement. D'autre part, parce que le législateur a coupé court à toute interrogation en donnant purement et simplement satisfaction au camp des adversaires de la jurisprudence.

Au final, au terme d'un débat parlementaire réduit à sa plus simple expression et sommairement tranché en commission mixte paritaire, on a écrit dans la loi : (1) qu'il faudrait désormais un lien de causalité directe entre la faute et le dommage pour que l'enfant puisse prétendre à l'indemnisation du préjudice ; (2) que les parents eux-mêmes ne pourraient pas accéder à la réparation de leur préjudice moral $^{7}$; bref, enfants et parents étaient invités à considérer leur mésaventure comme un regrettable accident de la nature et n'avaient en conséquence qu'à se tourner vers la solidarité nationale et les prestations forfaitaires de la protection sociale que l'on promettait plus généreuses à l'avenir.

\section{Socialiser la réparation?}

La question sociale aura été la grande absente de ce débat. Pouvait-on imaginer une socialisation du type de risques dont l'affaire Perruche portait l'illustration? Certains répondront qu'elle existait déjà puisque les mécanismes de la protection sociale permettent aux handicapés d'accéder à des prestations et à une prise en charge de leur handicap par la collectivité. Mais ce type de socialisation présente deux inconvénients majeurs : son insuffisance et sa généralité.

Son insuffisance : il est généralement admis par tous les acteurs du débat que les prestations prévues pour les handicapés n'offrent pas un niveau de garantie et de couverture satisfaisant. Sans entrer dans le détail des mécanismes fort complexes de ces allocations qui visent à garantir un minimum de moyens aux personnes concernées, il faut relever que I'AES (allocation d'éducation spéciale) offre une prestation de 107,17 € par mois pour les enfants dont le taux d'incapacité est supérieur à $80 \%$ et qui sont obligés d'avoir recours à l'aide quotidienne, mais discontinue, d'une tierce personne (catégorie 1). Son montant s'élève à 896,55 € par mois pour les enfants

7. Cette réforme, il faut le souligner, a consisté tout bonnement à créer pour certaines catégories de praticiens un régime de responsabilité dérogatoire au droit commun. atteints d'un handicap particulièrement grave justifiant des soins continus de haute technicité (catégorie 3). II faut souligner en outre que les allocations prévues pour les adultes handicapés (Allocation de compensation pour le recours à une tierce personne, Allocation adultes handicapés) peuvent être modulées en fonction des ressources du bénéficiaire et sont dépourvues de tout caractère indemnitaire.

Sa généralité : ce type de solidarité suppose ensuite une certaine banalisation des parcours individuels, une communauté de traitement entre les handicaps parfaitement accidentels ou imprévisibles et les autres. Elle implique un «voile d'ignorance » qui consolide le contrat social en « anonymisant » les responsabilités et en plaçant chacun dans une situation d'égalité théorique devant le risque et l'obscure élection du malheur. Traiter ainsi, de manière forfaitaire, des handicaps liés - même indirectement - à des fautes médicales, c'est nier une partie du récit désormais disponible, puisque, en l'occurrence, on sait partiellement par où le malheur est arrivé. Or, les victimes peuvent légitimement demander à ce que leur trajectoire personnelle soit reconnue dans sa singularité, parce qu'autre chose que le hasard y a pris part. La nouvelle puissance de la médecine prédictive appelle du récit et des explications où l'on ne voyait naguère qu'une succession accidentelle d'événements. Ces demandes sont moins le fruit d'une «société de réparation » qui n'accepterait plus «la venue au monde d'un être non conforme à une normalité décrétée », comme on a pu le lire sous la plume du Président du Conseil consultatif national d'éthique [4], que le résultat d'une société technique où certaines fatalités se transforment peu à peu en risques rationnels et où «la venue au monde » s'accompagne d'obligations sociales plus individualisées.

Faut-il pour autant faire reposer toute la responsabilité du préjudice sur une imputation individuelle ? Faut-il nécessairement un responsable sur qui faire peser tout le poids des réparations? Bref, y a-t-il un moyen terme possible entre une socialisation aveugle et sourde aux demandes de reconnaissance individuelles, d'une part, et une logique d'imputation judiciaire qui ne pense la réparation que dans le cadre d'un procès civil ? La réponse est oui : ce serait le pari d'une socialisation du risque par la constitution d'un fonds public d'indemnisation des victimes auquel pourraient contribuer collectivement les professions médicales concernées ${ }^{8}$.

8. Deux cents affaires analogues à l'affaire Perruche étaient en attente de jugement au moment où le législateur est intervenu. Si la moitié de ces affaires avaient été retenues par la Cour de cassation, le coût moyen des indemnisations accordées jusqu'alors ayant été d'environ $300000 €$ par affaire, nous nous serions trouvés à moyen terme face à un contentieux d'environ 38 millions d'euros. 
Il s'agirait bien d'une indemnisation et non seulement d'une protection sociale générale et neutre : une logique indemnitaire donc, mais détachée de sa seule formulation judiciaire, qui contribuerait à reconnaître l'existence d'une faute et d'une victime, mais renoncerait à en imputer les conséquences à une personne particulière, nécessairement faillible. Ce dispositif d'indemnisation aurait l'avantage de ne pas tout mélanger, de porter reconnaissance aux individus, de reconstruire du récit, mais en socialisant la réparation. Une telle logique permettrait de concilier la vulnérabilité du praticien et celle de la victime.

Dans la fièvre de la polémique, cette hypothèse n'a malheureusement pas été retenue, ni même examinée.

\section{Conclusions}

L'arrêt Perruche est le résultat d'une interprétation possible du droit. II relève d'une logique judiciaire dont il ne faut pas attendre des jugements de nature politique ou d'intérêt général. II ne crée pas un nouveau régime de causalité, ni un prétendu «droit de ne pas naître ». Mais il laisse en l'état une autre problématique dont la résolution passait certainement par une initiative politique réfléchie et de nouveaux dispositifs de socialisation du risque thérapeutique. Le législateur a préféré modifier la loi et inventer, pour les praticiens de la médecine prénatale, un régime de responsabilité dérogatoire aux principes du droit commun. Du même coup, la question sociale n'a jamais pu être posée correctement. Tout comme la réflexion juridique, elle aura fait les frais d'une inflation du discours éthique, luimême sous-tendu par des revendications catégorielles (celles des associations de handicapés qui ont contesté la jurisprudence) et professionnelles (celles des praticiens de la médecine prénatale et des échographistes en particulier).

La controverse autour de l'arrêt Perruche témoigne également de notre difficulté à appréhender l'objet juridique dans son champ de pertinence et à le distinguer d'autres logiques : logique politique, logique sociale, mais aussi logique morale. Le fait qu'un débat de nature quasi métaphysique vienne se greffer sur un tel objet devrait, du reste, nous interroger. La Cour de cassation devrait-elle devenir, faute d'autorités morales socialement acceptées, le Vatican d'un nouveau clergé judiciaire? Certainement pas : il faut se garder de la confusion des genres. La justice n'a pas vocation à dire le bien, mais le juste. Qu'une société en mal de références morales reporte sur elle le poids de son deuil ne doit pas la charger pour autant d'une responsabilité qui n'est pas la sienne. $\diamond$

\section{SUMMARY}

Looking back on jurisprudence in the Perruche's case For over a year, jurisprudence in the Perruche case was the focus of vehement critiques. Some stemmed from a lack of understanding of the law, its way of reasoning, and its nature; others concerned the social consequences of the judicial decision. A legal response is hereby proposed that challenges the first critiques on the basis of three points of debate: the regime of causality, the child's standing to sue, and the alleged « right not to be born ». In the second case, the response can only be political. What is defended here differs considerably from the position adopted by the legislature several months ago. A device is proposed that would individualize responsibility and socialize compensation, with an eye to reconciling the vulnerability of the medical practitioner with that of the victim. $\Delta$

\section{RéFÉRENCES}

1. Cayla 0, Thomas Y. Du droit de ne pas naître. Paris : Gallimard, 2002.

2. Lacub M. Le crime était presque sexuel. Paris : Epel, 2002.

3. Hermitte MA. Le contentieux de la naissance des enfants handicapés. La Gazette du Palais $1997 ; 2^{\mathrm{e}}$ semestre : 1045.

4. Sicard D. Une société de réparation. Le Monde 6 décembre 2001.
TIRÉS À PART

T. Pech 